\title{
Correction to: Gyral-sulcal contrast in intrinsic functional brain networks across task performances
}

\author{
Lin Zhao ${ }^{1,2} \cdot$ Tuo Zhang ${ }^{1} \cdot$ Lei Guo $^{1} \cdot$ Tianming Liu $^{2} \cdot$ Xi Jiang $^{3}$ \\ Published online: 4 January 2021 \\ (C) Springer Science+Business Media, LLC, part of Springer Nature 2021
}

Correction to: Brain Imaging and Behaviors (2020) https://doi.org/10.1007/s11682-020-00347-x

The original published version of this article contained mistakes. The corresponding authors should be Tuo Zhang and Xi Jiang.

Publisher's note Springer Nature remains neutral with regard to jurisdictional claims in published maps and institutional affiliations.

The online version of the original article can be found at https://doi.org/ $10.1007 / \mathrm{s} 11682-020-00347-\mathrm{x}$

Tuo Zhang

tuozhang@nwpu.edu.cn

$\triangle$ Xi Jiang

xijiang@uestc.edu.cn

1 School of Automation, Northwestern Polytechnical University, Xi'an, China

2 Cortical Architecture Imaging and Discovery Lab, Department of Computer Science and Bioimaging Research Center, The University of Georgia, Athens, GA, USA

3 School of Life Science and Technology, University of Electronic Science and Technology of China, Chengdu, China 